\title{
La calidad una lección aprendida de experiencia japonesa, para emular en las Mipymes
}

\author{
pags $127-141$ \\ Grupo de investigación: Centro de investigación en Desarrollo Empresarial. CINDE \\ Carlos Gabriel Correa Ch.•
}

Recibido: Abril 7 de 2014

Aceptado: 30 de mayo de 2014

\section{RESUMEN}

El artículo pretende mostrar la identificación de estrategias implementadas por la industria japonesa, con las cuales pudieron resurgir, después de haber sido impactadas en su economía e infraestructura al terminar la segunda guerra mundial, no obstante sus limitados recursos naturales, ser tercera parte de la extensión colombiana, haya logrado mantenerse como potencia exportadora de calidad mundial. Se reflexiona sobre concepto actual de calidad en un mundo globalizado con facilidades tecnológicas de comunicación en las relaciones comerciales entre productores y consumidores. Las estrategias implementadas se basaron en principios de calidad recomendados y enseñados por Edwards Deming, Joseph Juran y Armand Feigenbaum;estos principios y estrategias al implementarlas en mipymes colombianas, se espera alcanzar competitividad, fiabilidad, fidelidad y confianza hacia las empresas y sus productos

Palabras claves: calidad, fiabilidad, fidelidad, principios de calidad.

\begin{abstract}
The article shows the identification implemented by Japanese industry strategies with which they could resurface, after being impacted its economy and infrastructure after the Second World War, despite its limited natural resources and being only a third of the Colombian extension, has managed and maintained as a world-class exporter. It reflects on current concept of quality in a globalized world with communication facilities in the commercial relations between producers and consumers. Implemented strategies were based on principles recommended and taught by Edwards Deming, Joseph Juran and Armand Feigenbaum quality; principles and strategies implemented Colombian SMEs, is expected to achieve competitiveness, reliability, loyalty and trust companies and their products
\end{abstract}

Keywords: quality, reliability, accuracy, quality principles.

- Docente investigador Universidad de América, carlos.correa@profesores.uamerica.edu.co Centro de investigación y desarrollo empresarial CINDE, línea de investigación: Desarrollo de mipymes 


\section{INTRODUCCIÓN}

Las experiencia lograda en competitividad de calidad en un mundo globalizado por la industria japonesa al haberse transformado en una de las naciones más industrializada del mundo, un país productor de bienes y servicios de calidad mundial, reconocido por los consumidores, no obstante que sus limitantes en recursos naturales y los frecuentes desastres naturales, superados con resiliencia sin tener que acudir a ayudas externas; los resultados, un país que sigue siendo ejemplo a emular por la industria colombiana; un país rico en recursos naturales, pero con necesidades de empleo, no exportador de productos procesados con una baja producción, con la tendencia a ser más comercial que industrial, según cifras del DANE: a noviembre de 2013, 23 de los 44 subsectores industriales registraron disminución en su producción en un promedio de caída del $4.3 \%$, mientras que las ventas crecieron 5.3 \% (El Siglo, 2014)

Colombia ha suscrito tratados de libre comercio con países que tienen ventajas de recursos tecnológicos para la producción de artículos de consumo como resultado de procesos que agregan valor a materias primas muchas de ellas suministradas por este país, la tendencia de adquisición de maquinaria no es la de adquirir tecnología actualizada, por no decir de punta sino que se adquiere maquinara depreciada, que ya ha cumplido con su tiempo de trabajo, transformándose en un problema de productividad ocasionado por paradas de tipo mecánico o eléctrico y desperdicios de materias primas por desgaste de piezas que controlan características de calidad, como ha sido el caso del sector de artes gráficas

Se analizaron las causas y el efecto principal en las que los clientes cada vez más exigen calidad, definido generalmente como el grado en el cual un producto específico satisface los requerimientos de un cliente o consumidor, que el producto o servicio cumpla con lo esperado o estipulado en tiempo y lugar; los clientes, con los sistemas actuales de comunicación adquieren y buscan solución de sus necesidades no solo a nivel local, sino global; el uso masivo de los sistemas de comunicación han hecho un mundo en el que los tics reducen distancias medidos en términos de tiempo, estas son iguales; los clientes cuentan con más proveedores en el mismo instante, que ofrecen ventajas de alta competencia; la calidad es factor primordial en la decisión de compra y de fidelización de los clientes con las empresas proveedoras, la experiencia del Japón analizada desde los aportes de principios y teorías puestas en práctica por los gurús de la calidad Edwards Deming, J.M. Juran y Armand Vallin Feigenbaum hasta hoy cuando los clientes de sus productos a nivel global, tienen la seguridad de recibir productos de calidad.

La metodología desarrollada se realizó investigando la evolución industrial del Japón desde finales de la segunda guerra mundial en el año 1945, cuando dejo un completo desastre, daños y especialmente en la industria y al aceptar el apoyo de los estos expertos en el tema de la calidad iniciaron un proceso de reconstrucción y de pensamiento en grande en el que sus clientes no eran solo a nivel local sino a nivel mundial.

La finalidad y objetivos desarrollados corresponden a conocer las estrategias puestas en marcha en las empresas japonesas analizando los principios y teorías propuestas con la posibilidad de emularlas de manera practica en las empresas Pymes del país, contrastar los hábitos de los trabajadores y sistemas administrativos en las empresas colombianas

\section{JUSTIFICACCIÓN}

Las empresas desde pequeñas y medianas hacen frente hoy a una fuerte competencia no solo a nivel nacional, sino a nivel internacional, cuyas características del mercado hacen que tengan la necesidad de cumplir con parámetros en la satisfacción total del consumidor, por lo que la calidad se ha convertido en la clave de éxito en todos los negocios, asegurándola en todos los procesos tanto administrativos como opera- 
LÍNEA DE INVESTIGACIÓN: MIPYMES

tivos; la experiencia alcanzada por las empresas japonesas en poco tiempo justificó ahondar en buscar cuales fueron las estrategias para el logro adquirido

\section{HIPÓTESIS}

¿La experiencia de haber implementado los principios y teorías enseñadas por los gurús de la calidad Edwards Deming, J.M. Juran y Armand Vallin Feigenbaum, en las empresas japonesas con resultados de calidad reconocidas a nivel mundial, será posible implementarlas en empresas Pymes en Colombia y poder lograr resultados de competitividad similares?

\section{DESARROLLO}

\section{Antecedentes de la calidad}

La calidad ha sido una filosofía de hacer bien las cosas, teniendo en cuenta su uso y tiempo de disfrute esperado por el cliente, se tiene conocimiento de la existencia de normas, políticas y directrices con este propósito, cumplir con las expectativas de quien solicita un producto o un servicio; el origen de este concepto se conoce desde el Código de Hammurabi ${ }^{1}$, dio parámetros desde el año 1760 a.C, sobre la responsabilidad de calidad, al declarar en una de sus leyes

"Si un albañil construye una casa para un hombre, y su trabajo no es fuerte y la casa se derrumba matando a su dueño, el albañil será condenado a muerte".

Se tiene conocimiento sobre la práctica de la inspección de calidad por parte de los inspectores egipcios que comprobaban las medidas de los bloques de piedra con un pedazo de cordel. Los mayas ${ }^{2}$ también usaron este método.

1 Sexto rey de Babilonia, creo el código en el año 1760

a.C., como primer conjunto de leyes de la historia

2 Cultura prehispánica más prestigiosa de Centroamérica
Calidad, factor que todo comprador evalúa y hace reconocimiento al recibir un producto o servicio que cumpla con sus expectativas y percepciones, es lo que desea recibir a cambio de lo que paga; la calidad ha tenido evolución en el concepto, no es algo estático y determinado desde un solo punto de vista, lo que para una persona, un producto es de calidad para otro, el mismo producto no lo es; lo estrictamente claro es que es un factor de fortaleza para una empresa, cuyo objetivo es el trúput ${ }^{3}$, haciendo que las ventas sean frecuentes y repeticiones de continuas pedidos; lo más importante para una empresa u organización, es el producto, el cliente no le importa quién lo hizo, pero lo que si le importa y paga con gusto, y se transforma en un reiterativo cliente, es que el producto o servicio cubra la expectativa esperada, para el tiempo de uso o disfrute que él determina o espera

\section{Algunas ideas de calidad}

La interpretación de la calidad se ha clasificado en cinco grupos o enfoques desde diferentes percepciones

Enfoque trascendente: se conoce lo que es calidad, pero no se puede definir- "Creo que sencillamente la reconozco cuando la veo" (Guaspari, 1985); al preguntársele a un trabajador de la empresa japonesa MATSUSHITA ELECTRIC CO. Cómo define la calidad, respondió. "es un estado de espíritu". Esta visión de calidad es absoluta, universalmente reconocible, pero imposible de definir.

Enfoque de producto: la calidad se puede medir y corresponde a una variable concreta y precisa. Esta evaluación permite crear jerarquía o grado de calidad, ejemplo el peso que puede soportar un cable antes de que se fracture. Esta definición es ambigua ya que un cable con inferior resistencia mecánica puede ser igualmente útil en una aplicación menos exigente

3 Velocidad con que el sistema genera dinero a través de lo que se hace, es la real venta 
Enfoque de fábrica: las definiciones basadas en el usuario incorporan elementos subjetivos que no pueden ser cuantificados fácilmente. Las definiciones basadas en la fabricación centran la atención en el grado de conformidad de las características de un producto con respecto a las normas establecidas por los departamentos técnicos de la empresa, ya sea que estas especificaciones se elaboren en la empresa o que sean establecidas por un cliente o normas preestablecidas

Enfoque del valor: define a la calidad en términos de costos y precios. La calidad vista como la satisfacción del usuario a un precio razonable pretende limitar las especificaciones técnicas, en relación con un precio que está dispuesto a pagar. Un producto puede estar muy bien fabricado, pero si su precio es elevado imposibilita la compra y su uso, por lo tanto, este producto no interesa a muchos compradores

Enfoque de la durabilidad: antes de los años 50's la durabilidad era el principal factor para identificar un producto de calidad, el ejemplo de Volkswagen desde el pueblecito de FaIlersleben, a orillas del Canal de Mittelland, cuya responsabilidad principal mostraba gran preocupación por la calidad y la reducción de costos para un coche económico, alcanzable de adquirir por el pueblo alemán. "El Volksauto dará movilidad al país y será el transporte de las masas" afirmaba el Führer, apoyando el diseño soñado por Ferdinand Porsche

La economía basada en el trabajo manual, sin estandarización, como trabajo artesano, fue reemplazada en la Revolución Industrial ${ }^{4}$, por la industria manufacturera, surgió la producción a gran escala, con piezas y elementos intercambiables, donde se imponía la medición o comprobación de estandarización; el tiempo de producción se redujo con incremento en la cantidad; el control de la calidad de los productos,

4 Surgida en la Gran Bretaña a mitad del siglo XVIII y principios del XIX se le atribuía al capataz ${ }^{5}$ y directores de producción, su prioridad era cumplir con los plazos fijados para fabricación, más que por la calidad, podría perder su trabajo si no cumplía con las demandas de la producción, mientras que sólo recibiría una observación, si la calidad era inferior; se crearon sistemas de incentivos, basados en cantidades sin ponderar la calidad. La calidad se vio relegada por la cantidad, estos sistemas todavía a los años 80's se habían implementado en la industria, la calidad era solo responsabilidad del departamento de fabricación o más específicamente del departamento de calidad

El sistema industrial moderno comenzó a surgir a fines del siglo XIX en los Estados Unidos, donde Frederick Taylor ${ }^{6}$, suprimió la planificación del trabajo como parte de las responsabilidades de los trabajadores y capataces y la puso en manos de los Ingenieros Industriales, que se les conocía como Ingenieros de Métodos y Tiempos.

El sistema de línea de ensamble en movimiento, creado e implementado por Henry Ford, en la fabricación de autos incrementó el número de unidades por hora, en un turno de 8 horas no se alcanzaba a producir un carro, sólo un poco más de medio o sea un $64 \%$, pasando a 5,16 automóviles y se creó la responsabilidad de la calidad a los operarios de cada avance del proceso; esta división del trabajo ha permitido la creación y el progreso en el ámbito de la investigación, el desarrollo y la innovación (I+D+i)

El concepto de calidad estaba basado en una vida útil larga, en la durabilidad, esta misma política la retomo en Alemania; Ferdinand Porsche $^{7}$, diseñó un coche familiar de cuatro plazas,

5 Persona responsable de dirigir un equipo de operarios al que se le asigna la asignación de una tareas

6 Ingeniero mecánico y economista estadounidense, promotor de la organización científica del trabajo y es considerado el padre de la Administración Científica

7 Es la cabeza del diseño del Volkswagen, nació en 1875, desde niño enorme capacidad creativa para el diseño de carros 
LÍNEA DE INVESTIGACIÓN: MIPYMES

con motor refrigerado por aire capaz de alcanzar los $100 \mathrm{~km} / \mathrm{h}$ (Prew, 1993) y a un precio alcanzable por el mercado, idea que fue apoyada por Adolfo Hitler; Tanto para el automóvil modelo $T$ de Henry Ford y el Volkswagen Beetle, el concepto de Calidad seguía siendo la durabilidad

La responsabilidad en la calidad de la VoIkswagen de gran ejemplo, en los primeros años de producción (1930), la empresa le reconocía al comprador, un motor en huacal y 4 llantas con banda blanca, previendo que en el tiempo se presentara defecto de calidad de tipo mecánico

La empresa General Motors (GM), líder como mayor fabricante de automóviles en los Estados Unidos, fue superada por la Ford, tuvo que adoptar una gran estrategia operativa de cambios anuales de modelos, colores y mejoras constantes de su producto, superando lo artístico a la durabilidad en la calidad mecánica (McNichols, 1977)

\section{USO DE LAS HERRAMIENTAS DE CONTROL DE CALIDAD}

Alrededor del año 1940, se incluye en los procesos de producción en las empresas, el control estadístico de la calidad, instituyéndose en las empresas el departamento de ingeniería de inspección, basado en herramientas estadísticas propuestas por George Edwards Deming y Walter A. Shewhart, quienes mostraron gran interés y lideraron la socialización, enseñanza e implementación en el Departamento de Agricultura de los EE.UU, El tema tuvo una adopción rápida, en el año 1946, cuando se había terminado la segunda Guerra Mundial; Deming visitó Japón en 1947 como Consejero en técnicas de Muestreo para el Cuartel General del Comando Supremo de las Potencias Aliadas, bajo las órdenes del general Douglas McArthur ${ }^{8}$, un año antes en 1946 inicia operaciones la Unión Japonesa de Científicos e Ingenieros (JUSE), como

8 Militar Estadunidense, encargado de recibir la rendición japonesa y luego comando la misión de ayuda a este país entidad pionera de la calidad japonés, preocupados por la mala calidad del producto, reconocen el éxito de la capacitación impartida por Deming, su director Kenichi Koyanagi, invitó el 8 de marzo de 1950, al Dr. Deming, solicitándole dos objetivos, que el llamo como favores

\section{"Impartir a ingenieros, gerentes de planta e investigadores japoneses unos seminarios en Control de Calidad.}

Contribuir con un mensaje al número inaugural de la revista mensual que empezaría a publicar el JUSE denominada "Control Estadístico de la Calidad".

No obstante las dificultades de envió y recorrido en el proceso de correos de la época, el Dr. Deming contesto la invitación, el 22 de abril del mimo año o sea a los 45 días cortos, con una carta que reflejaba la imagen precisa de una persona humilde, comprometida y con alto sentido del servicio (Orozco, 2001)

En el inicio del programa de capacitación se mostraban los asistentes muy escépticos, eran conscientes de que las materias primas y materiales que recibían eran de muy mala calidad, por fuera de las especificaciones técnicas, la primera observación del Dr. Deming, fue la urgencia de trabajar con sus proveedores y que procuraran mejorar su instrumentación, decía "no necesitan recibir la basura que le llega pues nunca producirán calidad con esa basura"

\section{LA EXPERIENCIA APRENDIDA}

¿Cómo fue entonces, que se logró la transformación que conocemos?, se pueden recopilar en los 14 puntos conocidos como la base de su filosofía de la calidad de Deming, identificadas como las teorías de calidad del Dr. Deming, las que se analiza cada una de ellas y sirven para efectuar diagnóstico actual de las empresas mipymes en Colombia, con base en estas teorías se hizo encuesta a empresas y son la base de las observaciones del investigador 
Tabla 1. 14 Teorías de calidad del Dr. Deming

\begin{tabular}{|c|c|c|}
\hline Teoría & Descripción & Comentarios del investigador \\
\hline 1 & $\begin{array}{l}\text { Crear constancia en el propósito hacia el mejora- } \\
\text { miento de bienes y servicios, con el fin de llegar a } \\
\text { ser competitivos y de esta manera mantenerse en } \\
\text { los negocios y sostener los empleos }\end{array}$ & $\begin{array}{l}\text { Uno de los problemas en nuestras empresas es } \\
\text { la falta de constancia, se emprenden mejora- } \\
\text { mientos, no se mantienen. }\end{array}$ \\
\hline 2 & $\begin{array}{l}\text { Adoptar la nueva filosofía. Nos encontramos en } \\
\text { una nueva era económica, la administración occi- } \\
\text { dental, debe despertar ante este reto. Debe asu- } \\
\text { mir sus responsabilidades y tomar el liderazgo } \\
\text { para el cambio }\end{array}$ & $\begin{array}{l}\text { No existe un sistema de comunicación de con- } \\
\text { fianza entre los diferentes niveles jerárquicos, } \\
\text { que facilite el trabajo en equipo }\end{array}$ \\
\hline 3 & $\begin{array}{l}\text { Terminar con la dependencia sobre inspección } \\
\text { final al } 100 \% \text {, para lograr la calidad. Eliminar la } \\
\text { necesidad de hacer inspecciones en forma masi- } \\
\text { va, integrando la calidad en el momento de hacer } \\
\text { el trabajo }\end{array}$ & $\begin{array}{l}\text { En nuestro medio no existe el concepto del } \\
\text { verdadero valor agregado en la trazabilidad en } \\
\text { cada uno de los procesos. } \\
\text { Existe dependencia total hacia el departamento } \\
\text { de control de calidad (inspección final masiva) }\end{array}$ \\
\hline 4 & $\begin{array}{l}\text { Terminar la práctica de conceder negocios con } \\
\text { base en la etiqueta de precio. Minimizar el cos- } \\
\text { to total. Buscar un proveedor único para un cierto } \\
\text { artículo estableciendo una relación duradera ba- } \\
\text { sada en la lealtad y la confianza }\end{array}$ & $\begin{array}{l}\text { El factor más importante en decisiones de com- } \\
\text { pra, el que más prevalece es el factor precio. } \\
\text { Los proveedores siente un mal trato, no hay leal- } \\
\text { tad con el proveedor, por otros factores, las em- } \\
\text { presas cambian de proveedor no por calidad de } \\
\text { productos y del servicio, tiene gran influencia las } \\
\text { referencias de amistad y de precios: no se pre- } \\
\text { senta fidelización y lealtad con los proveedores }\end{array}$ \\
\hline 5 & $\begin{array}{l}\text { Mejorar constantemente, y para siempre, el sis- } \\
\text { tema de producción y servicio para favorecer la } \\
\text { calidad y la productividad y así reducir los costos }\end{array}$ & $\begin{array}{l}\text { Los programas de mejoramiento son de moda, } \\
\text { sin continuidad, el cumplimiento de requisitos } \\
\text { de la norma de calidad, es más cuestión de } \\
\text { cumplimiento en la documentación, más que en } \\
\text { la satisfacción del cliente }\end{array}$ \\
\hline 6 & $\begin{array}{l}\text { Instituir métodos modernos para la capacitación } \\
\text { usando la estadística }\end{array}$ & $\begin{array}{l}\text { Los programas de capacitación no son asisti- } \\
\text { dos, los trabajadores y empleados no le dan im- } \\
\text { portancia, y los jefes no participan; no obstante } \\
\text { solo el } 5 \% \text { de los empleados de las pymes tie- } \\
\text { nen nivel universitario y el conocimiento de otra } \\
\text { lengua extranjera es de tan sólo } 4 \% \text { (El Espec- } \\
\text { tador, 1012) }\end{array}$ \\
\hline 7 & $\begin{array}{l}\text { Establecer métodos modernos para la supervi- } \\
\text { sión, considerando que ésta pertenece al sistema } \\
\text { y es responsabilidad de la administración }\end{array}$ & $\begin{array}{l}\text { La supervisión es tomado como un cargo de je- } \\
\text { fatura, no de facilitador de soluciones de proble- } \\
\text { mas u oportunidades que se presentan en los } \\
\text { procesos }\end{array}$ \\
\hline
\end{tabular}


LÍNEA DE INVESTIGACIÓN: MIPYMES

\begin{tabular}{|c|c|c|}
\hline Teoría & Descripción & Comentarios del investigador \\
\hline 8 & $\begin{array}{l}\text { Disminuir el temor a la autoridad para que los em- } \\
\text { pleados puedan trabajar eficazmente }\end{array}$ & $\begin{array}{l}\text { Nada más preocupante para un trabajador, que } \\
\text { el jefe lo llame; lo relaciona inmediatamente a } \\
\text { un error a un llamado de atención, a un termina- } \\
\text { ción o suspensión de un contrato } \\
\text { La buena relación de los trabajadores con sus } \\
\text { jefes se refleja en el rendimiento y la productivi- } \\
\text { dad laboral, los colaboradores de las empresas } \\
\text { buscan que sus superiores sean líderes (Porta- } \\
\text { folio, 2008) }\end{array}$ \\
\hline 9 & $\begin{array}{l}\text { Romper las barreras entre departamentos, alen- } \\
\text { tar las soluciones a los problemas a través del } \\
\text { trabajo en equipo }\end{array}$ & $\begin{array}{l}\text { Los departamentos son islas, no hay comunica- } \\
\text { ción asertiva entre ellos, se trabaja en grupos } \\
\text { pero no en equipo }\end{array}$ \\
\hline 10 & $\begin{array}{l}\text { Eliminar los lemas, exhortaciones, metas numéri- } \\
\text { cas, cartelones para la fuerza de trabajo }\end{array}$ & $\begin{array}{l}\text { Los lemas traídos de otras culturas, no son bien } \\
\text { recibidas. }\end{array}$ \\
\hline 11 & $\begin{array}{l}\text { Eliminar los estándares que prescriban cuotas nu- } \\
\text { méricas. Usar métodos estadísticos para el mejo- } \\
\text { ramiento continuo de la calidad y la productividad }\end{array}$ & $\begin{array}{l}\text { Los resultados de estadística e indicadores de } \\
\text { fácil interpretación son parte de la involucración } \\
\text { del personal, de acuerdo a sondeo de encues- } \\
\text { ta las herramientas estadísticas de calidad, se } \\
\text { realizan planes de muestreo para aceptar o re- } \\
\text { chazar lotes de producción mediante tablas de } \\
\text { la military estándar, las gráficas de control por } \\
\text { variables y las de control por atributos no son de } \\
\text { frecuente uso }\end{array}$ \\
\hline 12 & $\begin{array}{l}\text { Romper las barreras que impiden al trabajador } \\
\text { sentir orgullo por su trabajo }\end{array}$ & $\begin{array}{l}\text { No se tiene la cultura de la responsabilidad y } \\
\text { sentido de pertenecía por los productos de la } \\
\text { empresa; no es raro encontrar trabajadores ha- } \\
\text { ciendo compra de los productos de consumo a } \\
\text { la competencia } \\
\text { A nivel nacional no se tiene tampoco el sentido } \\
\text { de pertenecía por el producto nacional, el códi- } \\
\text { go de barras EAN } 13^{1}, 770 \text {, no es factor de deci- } \\
\text { sión en la compra, ni siquiera los clientes tienen } \\
\text { conocimiento de esta clasificación por países }\end{array}$ \\
\hline 13 & $\begin{array}{l}\text { Instituir un vigorosos programa de educación y } \\
\text { capacitación, para mantener a la gente al día de } \\
\text { los nuevos desarrollos materiales, métodos y tec- } \\
\text { nología }\end{array}$ & $\begin{array}{l}\text { Los programas de capacitación, no se hacen } \\
\text { con base en superar las falencias y debilidades, } \\
\text { quienes asisten a programas especiales, no ha- } \\
\text { cen socialización y difusión. }\end{array}$ \\
\hline
\end{tabular}

$1 \quad$ Versión de codificación más difundida a nivel mundial 


\begin{tabular}{|c|l|l|}
\hline Teoria & \multicolumn{1}{|c|}{ Descripción } & \multicolumn{1}{c|}{ Comentarios del investigador } \\
\hline 14 & $\begin{array}{l}\text { Definir claramente el compromiso permanente de } \\
\text { la alta administración, hacia la calidad y producti- } \\
\text { vidad. La transformación es trabajo de todos }\end{array}$ & $\begin{array}{l}\text { No es contundente mostrar que las altas di- } \\
\text { recciones se involucran en la calidad desafor- } \\
\text { tunadamente el mayor interés es la búsqueda } \\
\text { de certificaciones mostrar la documentación de } \\
\text { protocolos y evidencias }\end{array}$ \\
\hline
\end{tabular}

Fuente: basada en Administración por causas Vs. Administración por efectos

Los 14 puntos o principios se pueden clasificar en tres categorías: constancia de propósito $(1,14)$, mejora continua (2.3.4.5.6.11.12.13) y trabajo en equipo $(7,8,9$ 10) (Yacuzzi, 2003)

La fama de los productos japoneses en el año 50 , era de una mala calidad y por lo tanto las empresas no podían competir a nivel mundial, el Dr. Deming les llamó fuertemente y vehemente la atención al decirles:

"Ustedes pueden producir calidad, ya que cuentan con un método para hacerlo. Han aprendido lo que es calidad, por tanto deben llevar a cabo una investigación del consumidor, mirar hacia el futuro y producir bienes que tengan mercado, dentro de varios años y mantenerse en los negocios". (Orozco, 2001)

Las materias primas y materiales que recibían los japoneses eran de muy mala calidad; al efectuar control estadístico de calidad en la recepción técnica, las especificaciones eran fuera de control, fuera de color, nada estaba correcto, sugirió el Dr. Deming, que se debería trabajar de manera coordinada con los proveedores, procurando mejorar su instrumentación, repetía de manera insistente "no necesitan recibir basura que les llega pues nunca producirán calidad con esa basura”.

La filosofía de la calidad basada en la gente dio resultados sorprendentes y se cumplió la predicción antes de lo previstos en cuatro años, cuando decía que captarían los mercados de todo el mundo en cinco años, el mundo demandaba de sus productos, Los Estados Unidos, al término de la segunda guerra, sin competencia alguna, eran los dueños de los mercados de carros, electrodomésticos, la administración
Tayloriana se desarrolló exitosamente, eran los dueños de los mercados y así también se lo creían, esta bonanza empezó de desplomarse, los productos japoneses empezaron a invadir y tomarse el mundo, los mismos norteamericanos empezaron a ver y dejarse atraer por los productos del Japón, con diseños y calidad y goce en el uso por parte de quienes lo adquirían.

El uso de las herramientas estadísticas ya no eran utilizadas al término de los procesos, donde el resultado de dicha inspección final, aparecían productos rechazados y productos conformes, los rechazados iban a reproceso y/o a chatarra; el nuevo sistema incluía el control estadístico en cada proceso de la cadena productiva, dándole valor e importancia en el proceso PHVA, en el "Hacer", donde estaba el ser humano con capacitación, con motivación del quien lo hace, cambiando el proceso bajo el concepto de Taylor a un nuevo concepto de Deming

Las herramientas estadísticas del Dr. Walter A. Shewhart complementadas por las gráficas propuestas por Joseph Juran para el control de la calidad en los procesos, desafortunadamente la primera dificultad para el seguimiento en el valor agregad, la falta de información seguida del análisis oportuno de los resultados para la toma oportuna de decisiones que lleven a productos y servicios que de verdad satisfagan las expectativas de los clientes

La experiencia presentada en el documento "Érase una vez una fábrica..." de John Guaspari, apoya la búsqueda de la calidad, no es la de tener el máximo de supervisión, en el que se genera cantidad de burocracia y la obligación se distancia por los dueños de los procesos que 
LÍNEA DE INVESTIGACIÓN: MIPYMES

dejando a los supervisores la causa de mala calidad, se llega en sus "Conversaciones conmigo mismo", seis reflexiones para lograr competitividad en calidad mundial; los clientes le dicen a la empresa que tienen problemas de calidad ipero ni siquiera saben qué es Calidad!(Guaspari: 1985), es necesario escuchar con humildad y sentimiento de lealtad con nuestros clientes

\section{La calidad es asunto de supervivencia}

2. La calidad puede ser gratuita, pero es bastante menos costosa que las alternativas.

3. La calidad es una tarea de todos. Pero es responsabilidad de la gerencia

4. La mayoría de los problemas de Calidad están incorporados en el sistema

5. El primer paso para mejorar el sistema es conseguir información confiable sobre lo que es necesario soluciona

6. El segundo paso para mejorar el sistema es pasar de un modo de pensar centrado en la supervisión a una centrado en la prevención (Guaspari, 1985)

Joseph Juran ${ }^{9}$, identifica que muchas empresas partían desde el punto de que la calidad cuesta y por lo tanto se disminuyen las ganancias y que muchas de las deficiencias de los productos y procesos tienen su origen en la mala planificación de la calidad del producto o servicio que se desarrolla.

Juran define la calidad como la ausencia de deficiencias que origina problemas con el cliente: retrasos en la entrega, fallas del producto en el momento de ser utilizado, facturas incorrectas, ordenes de trabajo incorrectas, cancelación de pedidos, entre otros, problemas que a diario se repiten actualmente en las empresas; con-

9 De origen Rumano, recordado como un experto de la calidad y la gestión de la calidad, llamado el padre de la calidad, es uno de los gurús de la calidad cluye que la calidad es "adecuación al uso", propone dar la mayor importancia a la planificación de toda actividad que se desarrolla en cada proceso; una gran enseñanza de Juran, es que en el proceso de mejoramiento de Deming denominado como PHVA, la planeación (P), es a lo que mayor importancia debe darse, contestando a las preguntas: qué es lo que se va hacer, cuál va hacer el uso que el cliente le va a dar, o sea qué espera el cliente del producto o servicio, cómo se va hacer (requerimientos técnicos y humanos), cuán lo espera el cliente y donde; de esta forma se evitará que se originen problemas que como lo afirmaba Juran, generalmente ocasionaban un $20 \%$ de las perdidas en las empresas, propone entonces una trilogía de procesos: planificación, control y mejora;

Para el control recomienda la implementación de las herramientas estadísticas de calidad planteadas por el Dr. Walter A. Shewhart (Control estadístico de procesos), el Dr. Deming ferviente impulsor del uso de dichas herramientas, detecta el mismo problema de hoy en día, la falta de información en los ámbitos productivos atenta contra el control y la toma de decisiones oportunas, era la primera dificultad para el seguimiento de los resultados del control de la calidad y era consciente de que la estadística por si sola sin análisis no se podría lograr los resultados esperados; era necesario escuchar al cliente, pues él define la calidad

Basado en las expectativas del cliente, la mejora de la calidad debe tener en cuenta las necesidades y expectativas del usuario final, que es en definitiva quien acepta o rechaza el producto, por lo que todos los esfuerzos que realiza el personal deben ser orientados hacia el logro de las expectativas planteadas en cada orden de pedido claramente identificadas las necidaes

Al igual que Deming, Juran escribió una serie de recomendaciones conocidas como los 10 pasos para la mejora de la calidad: 
Tabla 3. Los 10 pasos para la calidad de Juran

\begin{tabular}{|c|c|c|}
\hline ítem & Pasos propuestos & Comentarios del investigador \\
\hline 1 & $\begin{array}{l}\text { Crear la conciencia de la necesidad } \\
\text { y oportunidad de mejora }\end{array}$ & $\begin{array}{l}\text { Siempre todo proceso y por su puesto todo producto es factible } \\
\text { de mejorar en función de la satisfacción del cliente }\end{array}$ \\
\hline 2 & Fijar objetivos de la mejora & $\begin{array}{l}\text { No es posible mostrar o demostrar mejora, si no son con objeti- } \\
\text { vos cuantitativos, superados }\end{array}$ \\
\hline 3 & $\begin{array}{l}\text { Organizarse para alcanzar los } \\
\text { objetivos(establecer un consejo de } \\
\text { calidad) }\end{array}$ & $\begin{array}{l}\text { Identificar los problemas, seleccionar proyectos, designar equi- } \\
\text { pos y facilitadores }\end{array}$ \\
\hline 4 & Entrenar & $\begin{array}{l}\text { Las competencias se deben actualizar, y solo se logran con pro- } \\
\text { gramas de entrenamiento y formación }\end{array}$ \\
\hline 5 & $\begin{array}{l}\text { Llevar a cabo los proyectos para re- } \\
\text { solver problemas }\end{array}$ & $\begin{array}{l}\text { Todo problema debe solucionarse como un proyecto específico } \\
\text { donde se vaya creando el concepto de resiliencia }\end{array}$ \\
\hline 6 & Informar sobre el progreso & $\begin{array}{l}\text { La retroalimentación o feedback, de los avances origina empa- } \\
\text { tía y empoderamiento por el mejoramiento }\end{array}$ \\
\hline 7 & Proveer reconocimiento & $\begin{array}{l}\text { Todo ser viviente necesita de reconocimiento, las llamadas que } \\
\text { se hacen a los trabajadores y empleados no solo deben ser para } \\
\text { llamados de atención, deben ser también para reconocer mejo- } \\
\text { ramiento }\end{array}$ \\
\hline 8 & Comunicar los resultados & $\begin{array}{l}\text { La comunicación efectiva debe incluir la socialización de los re- } \\
\text { sultados que se obtienen, con relación a las relaciones con los } \\
\text { clientes }\end{array}$ \\
\hline 9 & Mantener marcadores & Los marcadores de avances y logros deben socializarse \\
\hline 10 & Mantener el impuso & $\begin{array}{l}\text { La mejora anual debe hacer parte de los sistemas normales de } \\
\text { la empresa }\end{array}$ \\
\hline
\end{tabular}

Fuente: basada en Administración por causas Vs. Administración por efectos de Orozco, F., Jiménez,

Para el seguimiento en el proceso de control de calidad, de acuerdo al tipo de producto y a sus variables de calidad identificada por las características exigidas por los clientes como factor de cumplimiento se implementan utilizando graficas tanto de tipo numérico por variables como de manera cualitativa o de atributos

\section{GRÁFICAS DE CONTROL PARA VARIABLES}

Las gráficas como herramientas estadísticas son básicas para el proceso de análisis y solu- ción de problemas de calidad, las dos primeras A y B Diagrama de Ishikawa (causa-efecto) y Diagrama de Pareto y la tabla military standard como plan de muestreo o aceptación de lotes, son las que con alguna frecuencia las pymes las usan, le siguen en uso las gráficas de promedios y rangos, las de control para atributos son las menos utilizadas; para identificar las causas y los efectos de problemas, es frecuente el trabajo en equipo usando la gráfica de Kaoru Ishikawa, la gráfica de árbol de problemas y la de Pareto (Kume, 1985) 
LÍNEA DE INVESTIGACIÓN: MIPYMES

Tabla 4. Gráficas de control para variables

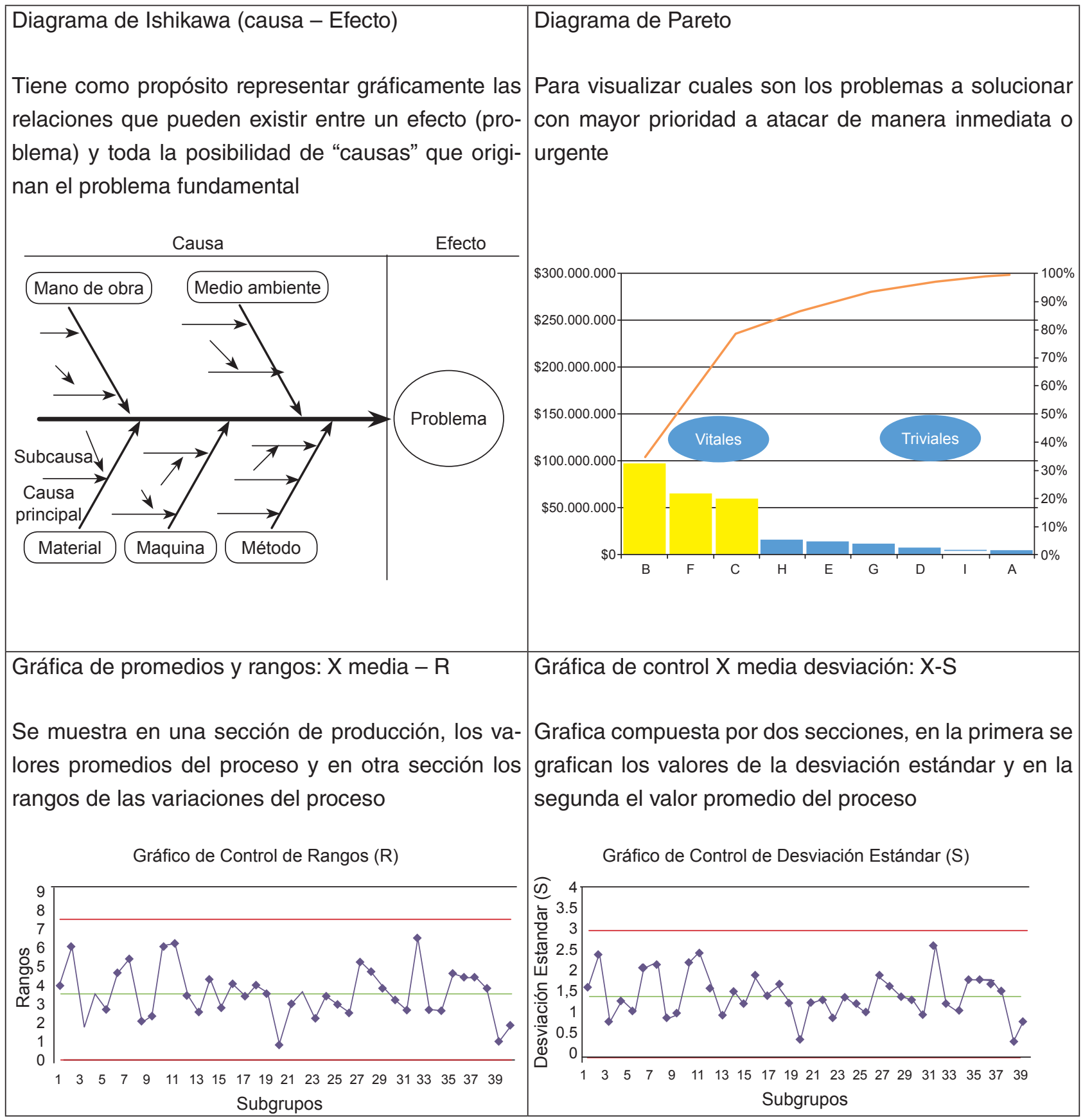

Fuente: Control Estadístico del Proceso, Angélica M. Soler Torres y Concepción López García, Management Developing Center MDC 


\section{GRÁFICAS DE CONTROL PARA ATRIBUTOS}

Cuando la característica de calidad no se puede medir o comparar con un patrón de medi- ción, como sucede con los datos para gráficas por variables, fueron planteadas otros tipos de gráficas de control conocida como gráficas por atributos

\section{Tabla 5. Gráficas de control para atributos}

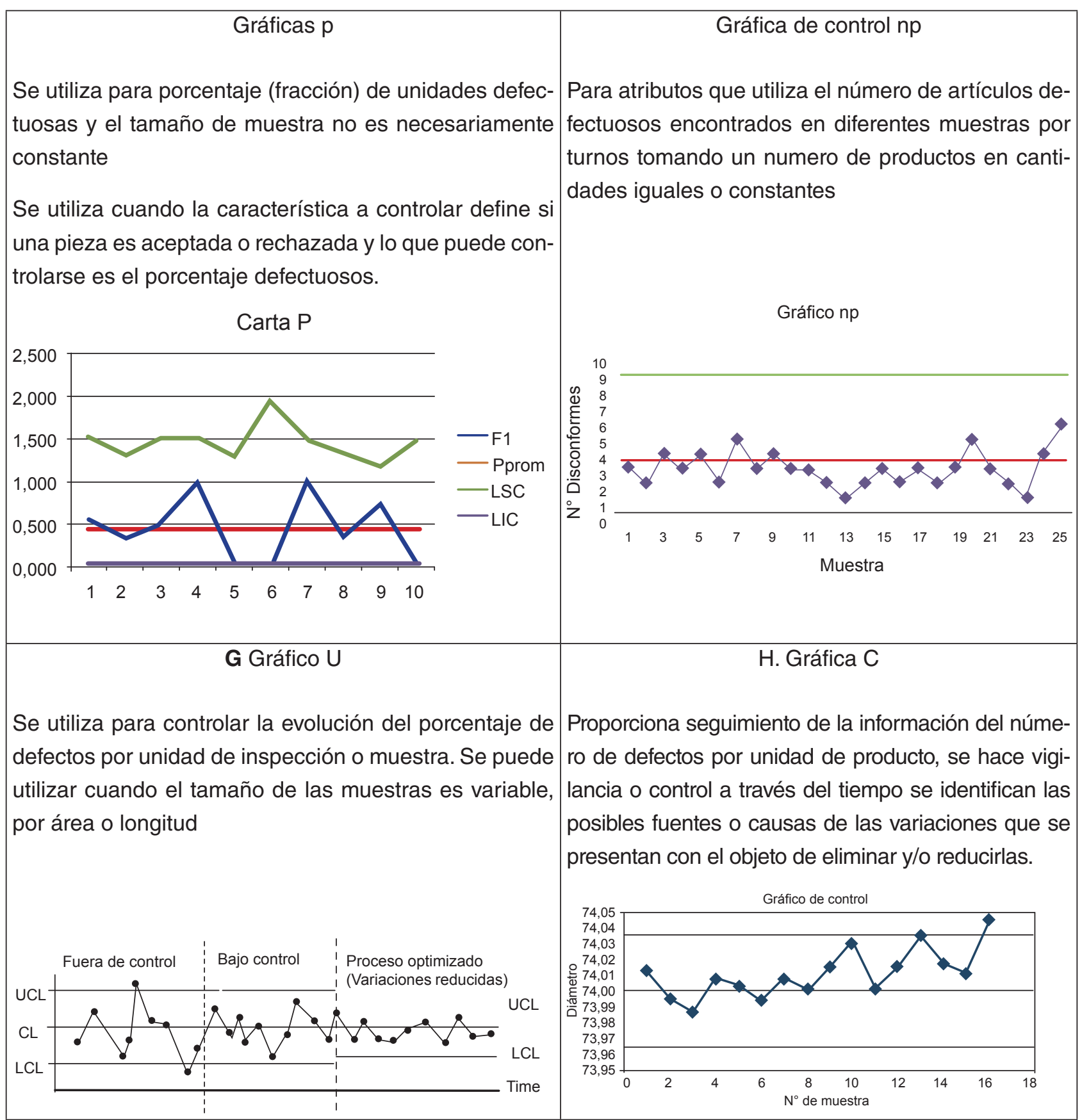

Fuente: Fuente: Control Estadístico del Proceso, Angélica M. Soler Torres y Concepción López García, Management Developing Center MDC 
LÍNEA DE INVESTIGACIÓN: MIPYMES

\section{CALIDAD TOTAL}

Armand V. Feigenbaum ${ }^{10}$, en 1960 fijó los principios básicos del control de la calidad total (Total Quality, TOC), en que se involucra toda la empresa desde el diseño hasta la postventa

“.. el tema desarrollado es, en definitiva hacer las cosas bien desde un principio. El énfasis está en la prevención de los defectos, reduciéndose la intensidad de la inspección de control. La calidad debe ser asegurada en cada una de las fases del desarrollo de un producto y con la colaboración de todos los trabajadores"

Fijó los principios básicos del control de la calidad total (Total Quality Control, TQC): el control de la calidad existe en todas las áreas de los negocios. Hasta ese momento todos los esfuerzos en la calidad habían estado dirigidos a corregir actividades, no a prevenirlas. Es así que en 1958, un equipo japonés de estudio de control de la calidad, dirigido por Kaoru Ishikawa, visitó a Feigenbaum en General Electric; al equipo le gusto el nombre TQC y lo llevó consigo al Japón, pero a una cultura muy diferente a la occidental especialmente en todos los niveles

En Colombia para un nuevo ambiente de competencia con tratados exigentes, hace necesario introducir en todas las organizaciones los principios de calidad basadas en las experiencias de numerosas empresas japonesas que no se quedaron en el que hacer, sino que fueron prácticos en la implementación con el objeto de poder competir en igualdad de condiciones, enseñanzas que además de aprenderlas se deben aprehender en las empresas Mipymes involucrando a los directivos dentro del proceso de gestión de la calidad

Se incluye uno de los aspectos más exigentes por los actuales clientes, que además de exigir las características técnicas de calidad, es

10 Empresario estadunidense y experto en control de calidad, diseñó el concepto de Control de la Calidad, luego conocido como Administración de calidad Total TQC el de la entrega a tiempo, el llamado Justo a tiempo (JIT), transformándose en factor de buena imagen en el mercado. Las reducciones del Lead Tome (tiempos de proceso total) reduciendo los tamaños de lotes y tiempo de aprovisionamiento exigiendo que el proveedor se integre con la empresa - cliente adaptándose a sus sistemas productivos y logísticos buscando mejores niveles de eficiencia conjunta, ya que cualquier presencia de defectos producirá paradas traumáticas en los equipos, como el sistema JIT, no acepta stocks que cubran estas paradas y se crean graves problemas en los plazos de entrega comprometidos con cada cliente. Las empresas y clientes no importando su tamaño exigen a sus proveedores participar de manera directa en los nuevos productos. Las empresas se transforman en proveedoras no solo con el reto de cumplir con las especificaciones, que debe ser normal, sino además en el tiempo acordado.

Con el sistema $\mathrm{TQC}^{11}$ y el $\mathrm{JIT}^{12}$, se puede lograr reducción de costos desde el mejoramiento de los procesos al interior del sistema de fabricación y al interior del proveedor, en la que la empresa que recibe los productos o materiales del ciclo productivo participa en la mejora de métodos reduciendo el consumo de recursos como materiales, buen uso de maquinaria, espacios, tiempos de producción, entre otros, obteniéndose un beneficio razonable que se le puede beneficiar al cliente final; es una cultura de beneficio propio de gana-gana

El concepto de calidad total o control de calidad total puede ser implementado en las Mipymes con resultados altamente favorables en la reducción de costos en todos los procesos desde la fase inicial como es el diseño, en el que los diseñadores tengan en cuenta con qué recursos técnicos, materiales, humanos y la misma infraestructura se cuente, integrando todas las áreas tanto administrativas como operativas

11 Control de la calidad total

12 Justo a tiempo 
y servicios externos como vigilancia y logística total (Yacuzzi, 2003)

Para lograr la calidad total se deben cumplir con tres requisitos fundamentales, señala Feigenbaum

- Hacer las cosas correctamente

- Hacer correctamente las cosas

- Hacer todo bien desde la primera vez

\section{MÉTODOS Y MATERIALES}

Se inicia con búsqueda de soluciones del problema de competencia por deficiencias de calidad, conociendo las recomendaciones de filosofía y de principios formuladas por los tres maestros de la calidad, para conocer cuáles fueron las estrategias implementadas para que este país Japón, haya alcanzado el éxito conocido, como una experiencia digna de aprender y de implementar. Con base en el conocimiento de los principios se hizo diseño de lista de chequeo tanto para ser diligenciado por personal de alta dirección y otro listado de encuesta dirigido a personal de supervisores; la consecución de disposición en lograr aceptación a las encuestas tuvo dificultad, no obstante se pudo recopilar aspectos que han servido para conocer aspectos de cumplimiento de dichos principios y poder observar las falencias en los procesos con el agregado de contar con calidad de productos sino además de la involucración del ser humano en todo el sistema integral de las empresas mipymes.

\section{CONCLUSIONES Y RECOMENDACIONES}

Las empresas mipymes, tienen un alto riesgo para poder competir a nivel global en lo relativo al compromiso de ofrecer productos de calidad a los clientes, las encuestas muestran un sondeo en que las respuestas son de tendencia positiva, pero en realidad la situación es necesario analizarla: desde el enfoque de jefes de área y supervisores se observa que es necesario un mayor compromiso en la capacitación del personal en temas referentes a calidad, el análisis de las causas que originan la pérdida de clientes deben ser prioridad, el personal de las empresas no muestran orgullo por los productos que se elaboran en la empresa, en las empresas y el uso de herramientas de estadísticas no es lo común en el proceso de control de calidad.

Se identificó las estrategias que desarrolló y ha desarrollado el Japón, experiencia para emularlas e implementarlas en empresas pequeñas y medianas (Pymes) que constituyen la principal fuente de empleo, y son parte fundamental del sistema económico.

Desde la percepción de la alta dirección, se observan algunas deficiencias que repercuten en la calidad de los productos y en la satisfacción de los clientes especialmente en los sistemas de comunicación al interior de las empresas, no es evidente que las empresas cuente o estén desarrollando con visión de futuro, planeación estratégica y si se tienen la socialización no es efectuada a todos los niveles

Las enseñanzas de Edwards Deming, Joseph Juran, Armand V. Feigenbaum, en la filosofía de calidad, en el uso de herramientas estadísticas para el control y en concepto de calidad total, dichas teorías basadas en primera instancia en la responsabilidad social al interior de las empresas al incluir la calidad de vida de sus trabajadores, debe ser implementada en todos los niveles de las empresas Mipymes.

En el documento no se incluye la evolución de los conceptos de calidad hacia el mejoramiento, como son las herramientas de Seis Sigma y Lean manufacturing, en razón a que no fueron planteadas en su época por los tres maestros de la calidad estudiados en el artículo 
LÍNEA DE INVESTIGACIÓN: MIPYMES

\section{BIBLIOGRAFIA}

Cantillo, D.C (5 de abril de 2014).Un país de Pymes. El Espectador, Economía, pp.4

Directivos distantes, empleados menos productivos, 2008, publicado en Portafolio 25 y 26 de octubre, p. 6

Guaspari, J. (1985). Un cuento sobre calidad Érase una vez una fábrica... Cali, Colombia: Editorial Norma S.A. (p. 31)

Guaspari, J. (1985). Un cuento sobre calidad Érase una vez una fábrica... Cali, Colombia: Editorial Norma S.A. (pp. 90 - 96)
Kume, H. (1985). Herramientas estadísticas básicas para el mejoramiento de la calidad. Bogotá, Colombia: Editorial Norma S.A.

Orozco, F., Jiménez, R (2001) Administración por causas Vs. Administración por efectos. México, D.F: Editorial Panorama

Prew, C (1993) Volkswagen-coche - historia, Madrid: Susaeta

Producción industrial no levanta cabeza, 2014, publicado en El Nuevo Siglo, 18 de enero, p.15 A

McNichols, T. (1977). Política empresarial con análisis de casos, Bogotá, Colombia: Editorial McGraw-Hill, p. 12-35

Moscoso, D. (16 de mayo de 2012). Los desafíos de las Pymes. El Espectador.

Yacuzzi, E. (2003). ¿Tiene relevancia la Gestión de calidad total? Reflexiones a la luz de las ideas de sus fundadores

Yacuzzi, E. (2003). ¿Tiene relevancia la Gestión de calidad total? Reflexiones a la luz de las ideas de sus fundadores. Recuperado de www. ucema.edu.ar/publicaciones/ dwnkoad/documentos/240.pdf 